\title{
A Novel Electric Dual Motor Transmission for Heavy Commercial Vehicles
}

\author{
Xiangyang $\mathrm{Xu}^{1,2}$. Jiajia Liang ${ }^{1,2}$ - Qingjun $\mathrm{Hao}^{3}$. Peng Dong ${ }^{1,2}$. Shuhan Wang ${ }^{1,2}$. Wei Guo ${ }^{1,2}$. Yanfang Liu ${ }^{1,2}$. \\ Zhonghua $\mathrm{Lu}^{3} \cdot$ Jiantao Geng ${ }^{3} \cdot$ Bin Yan $^{3}$
}

Received: 21 June 2020 / Accepted: 2 December 2020 / Published online: 11 January 2021

(C) The Author(s) 2021

\begin{abstract}
As a vital vehicle part, the powertrain system is undergoing a fast transition towards electrification. The new integrated electric drive system has been widely used, especially for passenger cars. In this work, a novel electric dual motor transmission is proposed for heavy commercial vehicles. The transmission scheme is firstly introduced, which can achieve 9 different operating modes including 5 single motor modes and 4 dual motor modes. Then, the mode shift map with minimum energy consumption is designed based on the motor efficiency map and the proposed energy management strategy. The driving power is appropriately distributed between the two motors in dual motor modes under the condition of minimum power consumption. In addition, a coordinated control strategy is developed for mode shift control without power interruption. The results show that the electric dual motor transmission has advantages in power consumption and power shift ability compared with the conventional single motor automated manual transmission.
\end{abstract}

Keywords Dual motor transmission · Operating mode $\cdot$ Mode shift map $\cdot$ Mode shift control $\cdot$ Torque distribution Coordinated control

$\begin{array}{ll}\text { Abbreviations } \\ \text { AMT } & \text { Automated manual transmission } \\ \text { C-WTVC } & \text { Adapted world transient vehicle cycle } \\ \text { eDMT } & \text { Electric dual motor transmission } \\ \text { EM } & \text { Electric motor } \\ \text { EMS } & \text { Energy management strategy } \\ \text { EV } & \text { Electric vehicle } \\ \text { MOP } & \text { Mechanical oil pump } \\ \text { PGP } & \text { Parallel gear pair } \\ \text { PTO } & \text { Power take-off }\end{array}$

Peng Dong

peng.dong@buaa.edu.cn

1 School of Transportation and Science Engineering, Beihang University, Beijing 102206, China

2 Ningbo Institute of Technology, Beihang University, Ningbo 315832, China

3 eKontrol Co., Ltd., Suzhou 215211, China

\section{Introduction}

The powertrain system plays a key role in the transition process of vehicle electrification. For passenger cars, it is not necessary to have a complex multi-speed transmission like traditional vehicles using the internal combustion engine (ICE) because the electric motor (EM) can provide maximum torque from zero speed and has a wide high-efficiency range [1]. Compared with the fixed-ratio reduction gear used widely in electric vehicles (EVs), a 2-speed transmission can improve the overall efficiency and dynamic performance of EVs [2-4]. Thus, the 2-speed electric transmissions for passenger cars have been developed and entered the EV market.

Following the footsteps of passenger cars, commercial vehicles have opened the door to electrification. Due to the complex operating conditions of heavy commercial EVs, multi-speed transmissions are still required. For example, trucks require large output torque off-road but high speed on road. The multi-speed transmission is thus mandatory. There are two types of electric drive, namely centralized drive and distributed drive [5]. The centralized drive has a mechanical differential that distributes torque evenly to the two-side wheels. The distributed drive means that the torque and speed of each wheel can be independently controlled by 
an EM. Since it is difficult to install a transmission for each EM of the distributed drive, the centralized drive is currently the main driving form of heavy commercial EVs.

Automated manual transmission (AMT) is widely applied in $\mathrm{EV}$ transmissions for commercial vehicles owing to its high efficiency [6]. However, the torque will be interrupted during the shifting process of AMT. Many researchers have conducted in-depth studies on the control methods to improve the shift quality [7-14]. Tseng et al. [15, 16] adopted AMT in an EV and proposed a gear-shifting control strategy including speed synchronization control and motion control of shift actuator. Gao et al. [17] used feedforward and feed-back controls for the clutch and the motor to achieve a smooth shift without torque holes. Mo et al. [18, 19] developed a novel synchronizer "Harpoon-Shift" as an alternative to the traditional cone clutch synchronizer aiming at improving comfort and efficiency. A shifting control method based on a special step function was proposed to improve shift comfort of AMT. Roozegar et al. [20] investigated the gear shifting of a multi-speed transmission for EVs with optimum performance under functional constraints. Three polynomials were proposed as transition functions of the angular velocities. Chen et al. [21] built a hybrid automaton model of the gear-shifting process in the clutchless coupled system using multi-body dynamics and hybrid system theories. By actively controlling the motor torque and shifting force, the clutchless coupled system could offer a better shift quality than systems with clutches. Wang et al. [22] proposed a position and force switching control scheme for gear engagement of AMT. The position controller was designed using the sliding mode control method. Simulation and test results showed that gear-shifting quality was improved, and gear-shifting shock was reduced greatly. In short, although the active speed regulation of the EM can shorten the shifting time, the AMT-based electric drive system cannot solve the problem of torque interruption in the shifting process.

The dual motor transmission has the power shift capability by maintaining one EM always in gear in the shifting process. Additionally, it can achieve a better operating efficiency along driving cycles by appropriate torque distribution and high torque utilization of the EM when using two downsized EMs to replace one EM with large power. Therefore, it is currently a research hotspot of the electric drive system. Wu et al. [23] compared the energy efficiency of dual motor transmission with that of single motor transmission. Results showed the advantages of dual motor transmission in overall efficiency along driving cycles. Based on the efficiency maps of two EMs, an energy management strategy (EMS) was developed for dual input power-split transmission [24] and dual input AMT [25]. Several driving cycles have been used to verify the effectiveness of the proposed EMS and demonstrate the improvement of energy efficiency using two motors. In addition, energy management strategies were also used in hybrid vehicles [26]. Sorniotti et al. [27] presented a novel clutchless seamless 4-speed transmission driven by two EMs. Compared with the single-speed and 2-speed transmission, it has better performance in terms of energy consumption and vehicle acceleration. Carlo et al. [28] combined two EMs through a planetary gear train, which makes EMs more efficient than a single motor. Zhang et al. [5, 29-31] proposed a new mode switching control method based on a dual motor centralized and distributed coupling drive system, which can reduce the mode switching shock. Thus, EV dynamic performance can be improved. Liang et al. [32,33] investigated the power shift capability of a dual motor AMT on EVs. The proposed shift control strategy took advantage of the dual motor transmission scheme, which greatly improved shift comfort. The above studies have clarified that dual motor transmission shows great potential in improving energy efficiency and power shift capability. However, they are mainly concentrated on passenger cars. There is still no research on dual motor transmissions for heavy commercial EVs.

The main contribution of this work is that a novel electric dual motor transmission (eDMT) is developed for heavy commercial vehicles. Based on the motor efficiency map, an EMS is proposed to determine the optimal mode and power distribution between the two EMs with minimal energy consumption. During the mode-shifting process, two EMs are coordinated and controlled to achieve a smooth shift without torque interruption. Overall, the novel eDMT aims to reduce energy consumption and eliminate shift interruption, thereby improving the energy efficiency and drivability of heavy commercial EVs.

The remaining sections of this paper are organized as follows. Section 2 introduces the scheme and operating modes of eDMT. In Sect. 3, the developed EMS and the mode shift map are discussed. Section 4 proposes the coordinated control strategy for mode shift control. Section 5 presents a case study and the corresponding simulation results. Finally, the conclusions are presented in Sect. 6.

\section{Transmission Scheme of eDMT}

Figure 1 presents the transmission scheme of eDMT, which adopts a modular design concept. It consists of two motors, namely EM1 and EM2. The mechanical oil pump (MOP) and power take-off (PTO) unit are driven by EM2 or EM1. Two shifting elements, A and CU, are dog clutches. They are actuated by a single shift drum with two forks, which is driven by a small actuator motor. The auxiliary gearbox is designed for torque amplification and is suitable for heavyduty vehicles of $90,000 \mathrm{~kg}$. For heavy vehicles of $31,000 \mathrm{~kg}$, no auxiliary gearbox is required. 


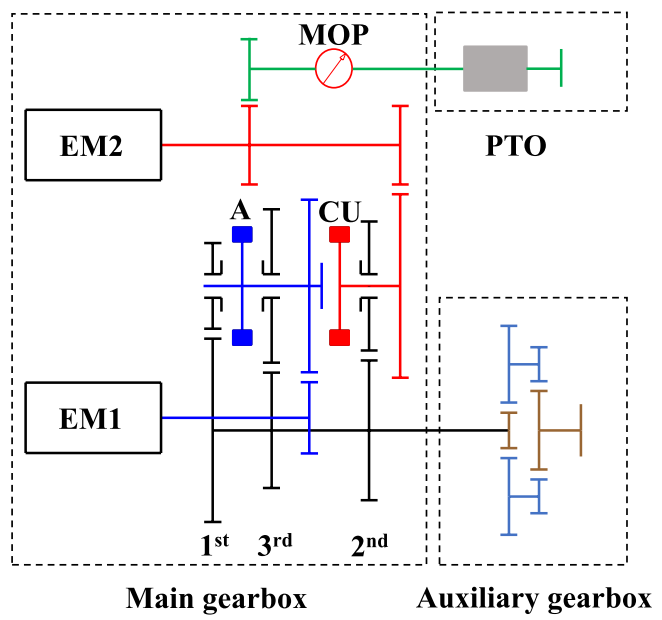

Fig. 1 The transmission scheme of eDMT

Figure 2 shows the operating modes of eDMT. There are 9 operating modes, including 5 single motor modes and 4 dual motor modes. Among them, mode M0 and M0.5 are specially designed for PTO output. In mode M0, the vehicle stops, and EM2 drives PTO to work. In mode M0.5, EM1 drives the vehicle to run slowly, and EM2 drives the PTO to work.

From mode M1 to mode M4, the gear ratio decreases and the single motor mode and dual motor mode appear alternately. The maximum output torque is obtained in mode M1 while the vehicle top speed is achieved in mode M4. It is apparent from the mode shift map (Fig. 3) that the eDMT offers a power shift capability because the single motor mode can be a transient transition mode during the mode

\begin{tabular}{|c|c|c|c|c|c|c|c|c|c|}
\hline \multirow{2}{*}{ Modes } & \multirow{2}{*}{$\begin{array}{c}\text { Power } \\
\text { sources }\end{array}$} & \multicolumn{3}{|c|}{ Dog clutch A } & \multicolumn{3}{|c|}{ Dog clutch CU } & \multicolumn{2}{c|}{ Gear Ratio } \\
\cline { 5 - 10 } & & L & M & R & L & M & R & EM1 & EM2 \\
\hline M0 & EM2 & & $\bullet$ & & & $\bullet$ & & $/$ & $/$ \\
\hline M0.5 & EM1 & $\bullet$ & & & & $\bullet$ & & 8.4 & $/$ \\
\hline M1 & EM1+EM2 & $\bullet$ & & & $\bullet$ & & & 8.4 & 13 \\
\hline M1.5 & EM1 & $\bullet$ & & & & $\bullet$ & & 8.4 & $/$ \\
\hline M2 & EM1+EM2 & $\bullet$ & & & & & $\bullet$ & 8.4 & 5.1 \\
\hline M2.5 & EM2 & & $\bullet$ & & & & $\bullet$ & $/$ & 5.1 \\
\hline M3 & EM1+EM2 & & & $\bullet$ & & & $\bullet$ & 1.7 & 5.1 \\
\hline M3.5 & EM1 & & & $\bullet$ & & $\bullet$ & & 1.7 & $/$ \\
\hline M4 & EM1+EM2 & & & $\bullet$ & $\bullet$ & & & 1.7 & 2.7 \\
\hline
\end{tabular}

L: Left position; M: Middle position; R: Right position; @: Engaged

Fig. 2 The operating modes of eDMT

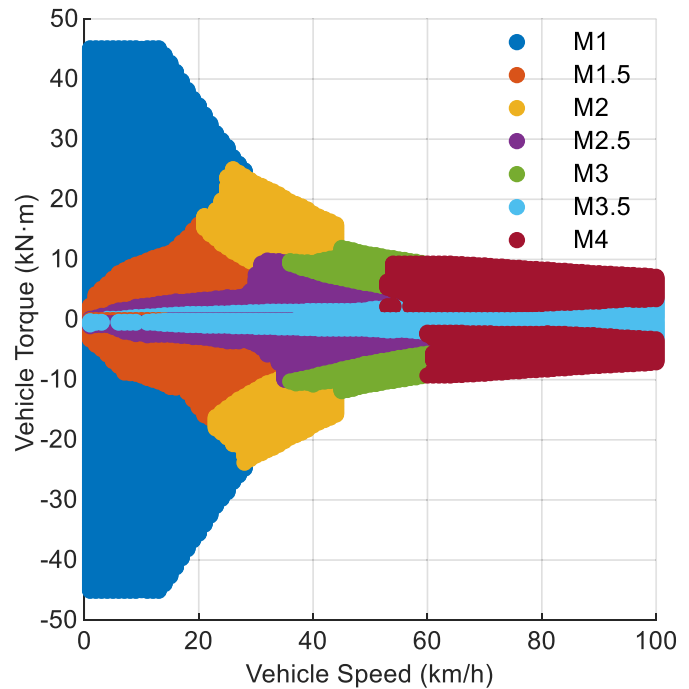

Fig. 3 The mode shift map of the eDMT with minimum energy consumption

shift. There is always one EM providing traction force to the wheels in the shifting process. For example, clutches A and $\mathrm{CU}$ are in the left position in mode M1. Both EMs drive the wheels through the 1st parallel gear pair (PGP). From mode M1 to mode M2, EM1 continuously drives the wheels through the 1st PGP. EM2 is shifted from the 1st PGP to the 2nd PGP by moving the dog clutch CU from the left position to the right position. The model M1.5 can be a transient transition mode during mode shift from M1 to M2, which can also be used as a steady single motor mode.

\section{Energy Management Strategy and Mode Shift Map}

\subsection{Energy Management Strategy}

To minimize the energy consumption of eDMT, a rule-based EMS is proposed to design the mode shift map. Since the eDMT has two EMs, another task of the EMS is to determine the power distribution between both EMs in dual motor modes.

For a specific vehicle operating point $\left(T_{\text {vehicle }}, v_{\text {vehicle }}\right)$ in the driving mode, the consumed power can be calculated as follows:

$P_{\text {discharge }}=\frac{T_{\mathrm{EM} 1} \cdot \omega_{\mathrm{EM} 1}}{\eta_{\mathrm{EM} 1}\left(T_{\mathrm{EM} 1}, \omega_{\mathrm{EM} 1}\right)}+\frac{T_{\mathrm{EM} 2} \cdot \omega_{\mathrm{EM} 2}}{\eta_{\mathrm{EM} 2}\left(T_{\mathrm{EM} 2}, \omega_{\mathrm{EM} 2}\right)}$

where $P_{\text {discharge }}$ is the discharging power from the battery, $\mathrm{kW} ; T_{\mathrm{EM} 1}$ and $T_{\mathrm{EM} 2}$ are the torque of EM1 and EM2, respectively, and the units are $\mathrm{N} \cdot \mathrm{m} ; \omega_{\mathrm{EM} 1}$ and $\omega_{\mathrm{EM} 2}$ are the angular velocity of EM1 and EM2, respectively, rpm; $\eta_{\mathrm{EM} 1}$ and 
$\eta_{\mathrm{EM} 2}$ are the operating point efficiency of EM1 and EM2, respectively.

Considering regenerative braking, EM1 and EM2 work as generators. The total charging power is expressed as follows:

$$
\begin{aligned}
& P_{\text {charge }}=T_{\mathrm{EM} 1} \cdot \omega_{\mathrm{EM} 1} \cdot \eta_{\mathrm{EM} 1}\left(T_{\mathrm{EM} 1}, \omega_{\mathrm{EM} 1}\right) \\
& +T_{\mathrm{EM} 2} \cdot \omega_{\mathrm{EM}} \cdot \eta_{\mathrm{EM} 2}\left(T_{\mathrm{EM} 2}, \omega_{\mathrm{EM} 2}\right)
\end{aligned}
$$

where $P_{\text {charge }}$ is the charging power to the battery, $\mathrm{kW}$. In Eqs. (1) and (2), the angular velocity of both EMs is dependent on the vehicle speed and the gear ratio of the operating mode, which is defined by the following equation:

$\left\{\begin{array}{l}\omega_{\mathrm{EM} 1}=\frac{v_{\text {vehicle }}}{r_{\mathrm{W}}} \cdot i_{\mathrm{EM} 1} \\ \omega_{\mathrm{EM} 2}=\frac{v_{\text {vehicle }}}{r_{\mathrm{W}}} \cdot i_{\mathrm{EM} 2}\end{array}\right.$

where $v_{\text {vehicle }}$ is the vehicle speed, $\mathrm{km} / \mathrm{h} ; r_{\mathrm{W}}$ is the dynamic tire radius, $\mathrm{m} ; i_{\mathrm{EM} 1}$ and $i_{\mathrm{EM} 2}$ refer to the gear ratios of corresponding operating modes. In single motor modes, the gear ratio of the non-operating EM is equal to 0 .

The vehicle torque demand or regenerative braking torque can be satisfied by both EMs in different operating modes, which imposes a constraint on the output torque of the two EMs by the following equation:

$$
T_{\text {vehicle }}=\left(T_{\mathrm{EM} 1} \cdot i_{\mathrm{EM} 1} \cdot \eta_{1}+T_{\mathrm{EM} 2} \cdot i_{\mathrm{EM} 2} \cdot \eta_{2}\right) \cdot i_{\mathrm{FD}} \cdot \eta_{\mathrm{FD}}
$$

where $T_{\text {vehicle }}$ denotes the torque demand or regenerative braking torque, $\mathrm{N} \cdot \mathrm{m} ; \eta_{1}$ and $\eta_{2}$ refer to the efficiency between EM and transmission output; $\eta_{\mathrm{FD}}$ is the efficiency of final drive; $i_{\mathrm{FD}}$ is the ratio of the final drive.

The objective of the proposed EMS is to minimize the discharging power or maximize the charging power for a specific vehicle operating point $\left(T_{\text {vehicle }}, v_{\text {vehicle }}\right)$. Equations (1)-(3) indicate that there are 4 independent variables, namely $i_{\mathrm{EM} 1}, i_{\mathrm{EM} 2}$, $T_{\mathrm{EM} 1}$, and $T_{\mathrm{EM} 2}$, among which the values of $i_{\mathrm{EM} 1}$ and $i_{\mathrm{EM} 2}$ are determined by operating modes of eDMT. The values of $T_{\mathrm{EM} 1}$ and $T_{\mathrm{EM} 2}$ are determined directly when the operating mode is a single motor mode. Otherwise, they will be globally traversed and compared to find the optimal values in the dual motor mode. It is assumed that PTO does not output torque. Therefore, the objective function can be expressed as minimum $P_{\text {discharge }}\left(i_{\mathrm{EM} 1}, i_{\mathrm{EM} 2}, T_{\mathrm{EM} 1}, T_{\mathrm{EM} 2}\right)$ or maximum $P_{\text {charge }}\left(i_{\mathrm{EM} 1}, i_{\mathrm{EM} 2}\right.$, $\left.T_{\mathrm{EM} 1}, T_{\mathrm{EM} 2}\right)$ subject to the following constraints:

$$
\left\{\begin{array}{l}
T_{\text {vehicle }}=\left(T_{\mathrm{EM} 1} \cdot i_{\mathrm{EM} 1} \cdot \eta_{1}+T_{\mathrm{EM} 2} \cdot i_{\mathrm{EM} 2} \cdot \eta_{2}\right) \cdot i_{\mathrm{FD}} \cdot \eta_{\mathrm{FD}} \\
\left|T_{\mathrm{EM} 1}\right| \leq T_{\mathrm{EM} 1 \_ \text {max }} \\
\left|T_{\mathrm{EM} 2}\right| \leq T_{\mathrm{EM} 2 \_\max } \\
\omega_{\mathrm{EM} 1} \leq \omega_{\mathrm{EM} 1 \_\max } \\
\omega_{\mathrm{EM} 2} \leq \omega_{\mathrm{EM} 2 \text { max }}
\end{array}\right.
$$

The enumeration method is applied in the optimization process. Firstly, all operating modes are enumerated orderly in the main loop. In the nested sub-loop, the output torque of EM1 is discretized by an interval of $10 \mathrm{~N} \cdot \mathrm{m}$ in the dual motor mode. The objective function will be calculated for each discrete point under the above constraints. Finally, the optimal operating points of both EMs and the optimal operating modes of eDMT are obtained for a specific vehicle operating point $\left(T_{\text {vehicle }}, v_{\text {vehicle }}\right)$.

\subsection{Mode Shift Map}

The mode shift map of the eDMT with minimum energy consumption is designed by adopting the proposed EMS, as shown in Fig. 3. In the figure, M0.5 is included in M1.5 because they are the same working mode. Moreover, the power distribution of driving condition and braking condition between both EMs in the dual motor modes is presented in Figs. 4, 5, 6, 7. Then, the minimum energy consumption at each moment can be calculated by combining Figs. 3, 4, $5,6,7$ and the transmission ratios.

\section{Coordinated Control Strategy for Mode Shift Control}

As mentioned above, one advantage of the eDMT lies in its power shift capability without torque interruption and thus the shift quality can be improved. However, it requires good control of the mode-shifting process. Therefore, a coordinated control strategy is developed in this section.

The proposed control strategy is explained according to the mode shift from M1 to M2 in Fig. 8. There are three phases. The function of each phase is summarized as follows:

Phase 1: Torque phase When the shift starts, the target mode is changed to M1. The torque of EM2 decreases gradually to unload the dog clutch CU. Meanwhile, the torque of EM1 is increased to compensate for the torque loss of the wheel. The wheel torque is thus transferred from EM2 to EM1, but the total wheel torque is the same as that before the shift. It is similar to the torque phase of a power shift with multi-plate wet clutches. However, the torque transferring is more accurate because of the precise control of EM torque.

Phase 2: Inertia phase When the torque of EM2 is decreased to 0 , the torque phase ends and the inertia phase starts. The dog clutch CU disengages firstly from the left position to the middle position, enabling the speed control of EM2. In this phase, the torque of EM1 is held at a constant level. The speed of EM2 is regulated from the current mode to the target mode. When the speed difference between the EM2 and the target mode drops into a predefined small 

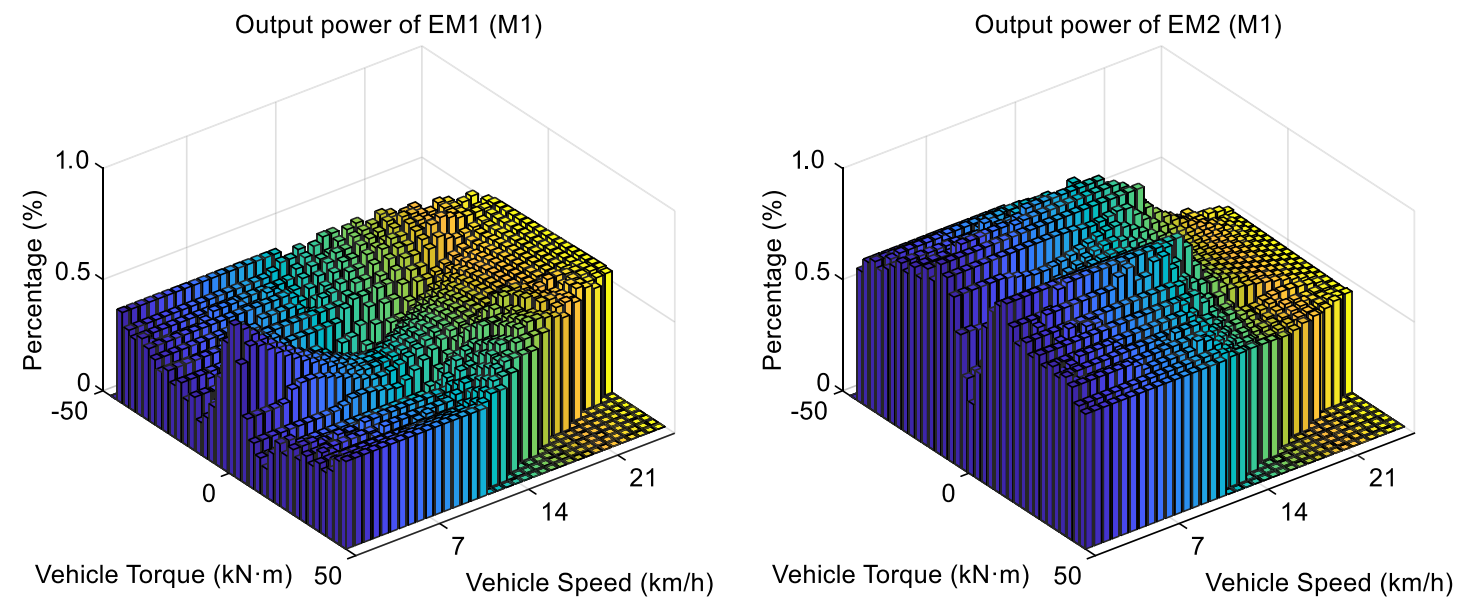

Fig. 4 Power distribution between both EMs in Mode M1
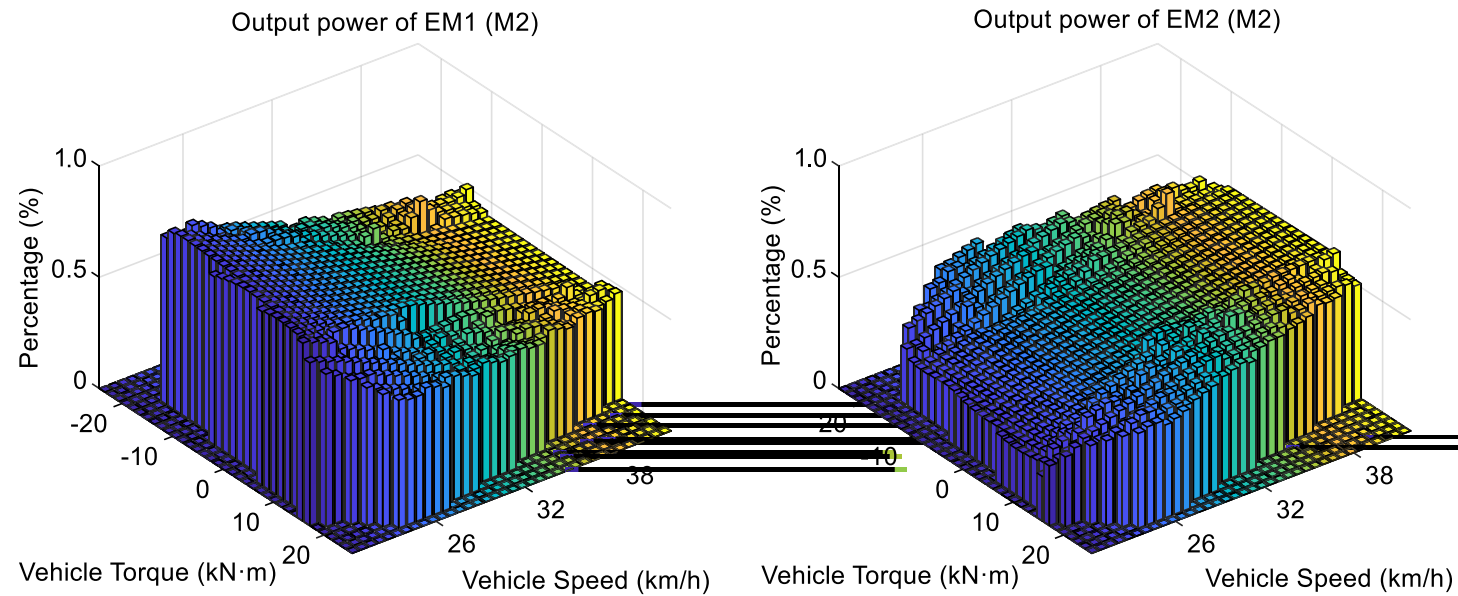

Fig. 5 Power distribution between both EMs in Mode M2
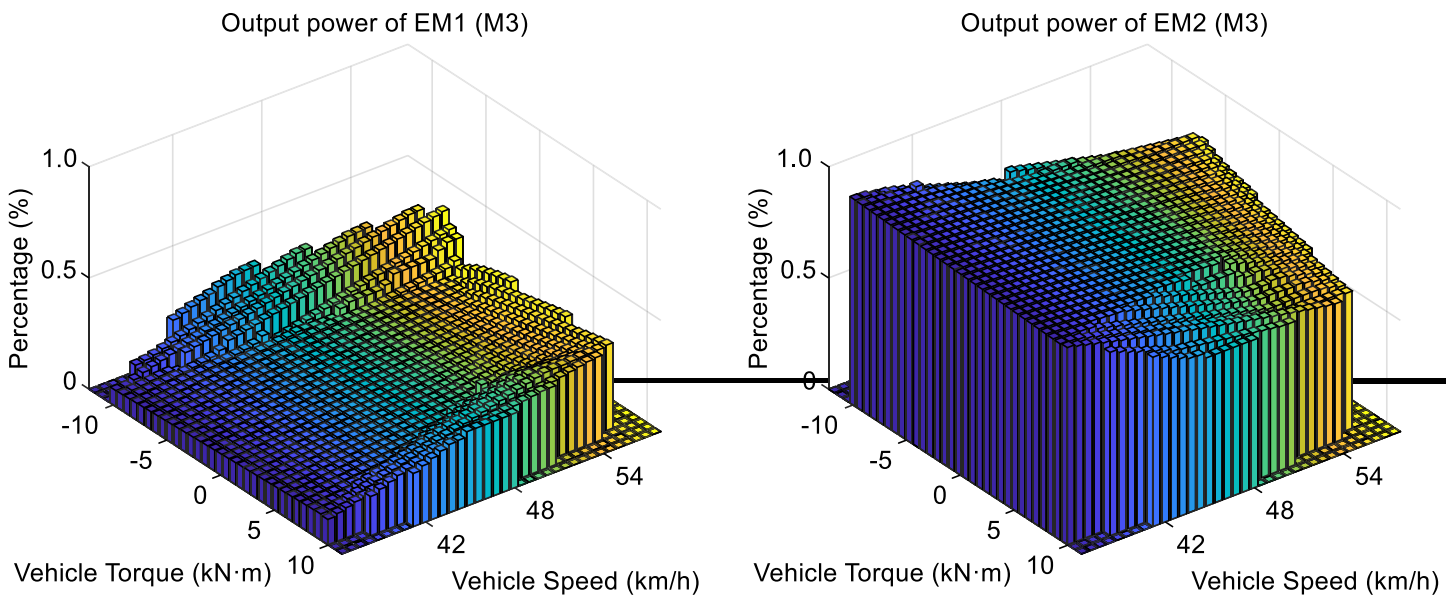

Fig. 6 Power distribution between both EMs in Mode M3 

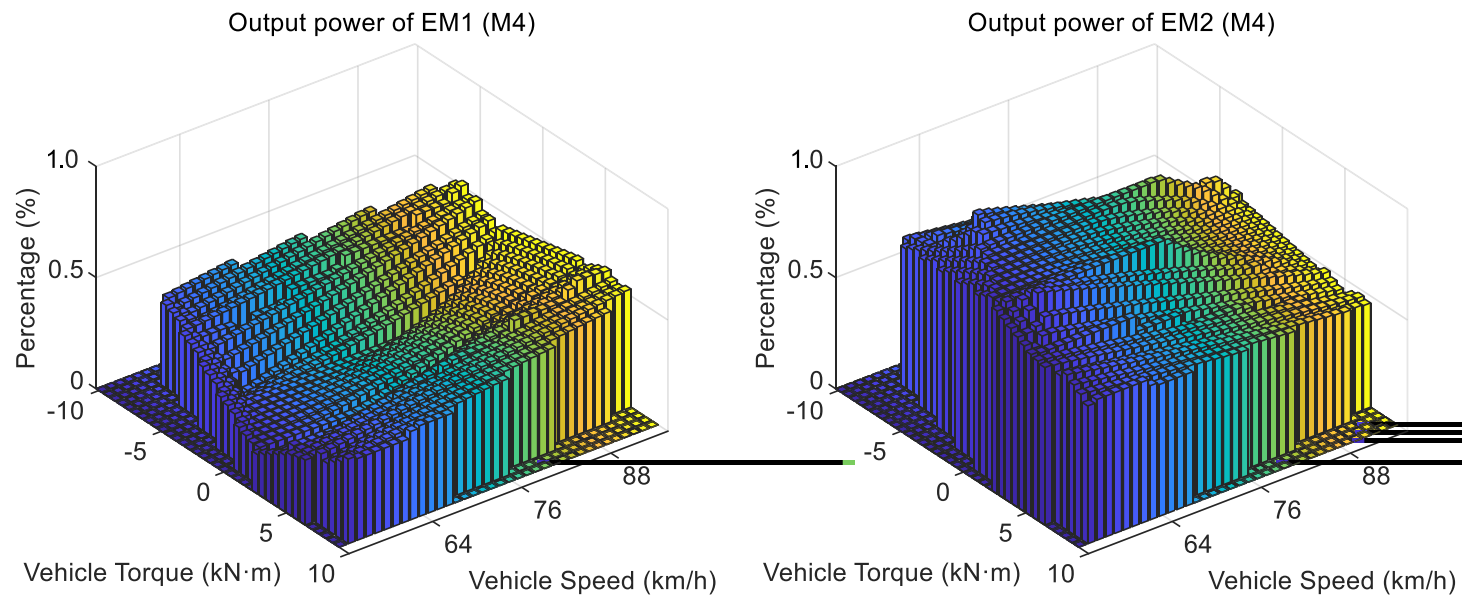

Fig. 7 Power distribution between both EMs in Mode M4

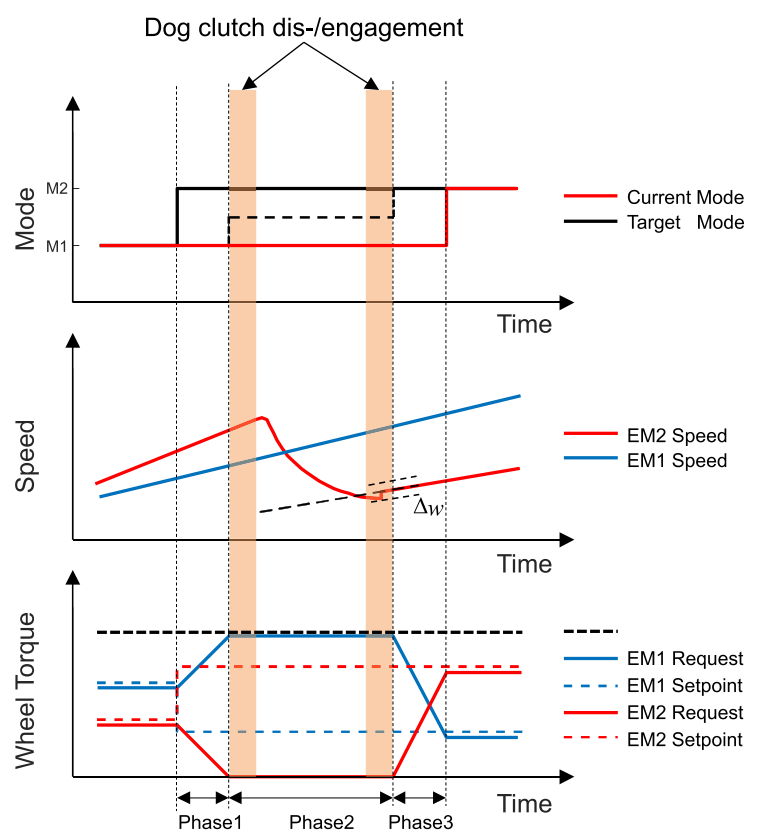

Table 1 Main parameters of the vehicle for simulation

\begin{tabular}{ll}
\hline Parameter & Value \\
\hline Vehicle mass & $31,000 \mathrm{~kg}$ \\
Wheel dynamic radius & $0.525 \mathrm{~m}$ \\
Frontal area & $10.5 \mathrm{~m}^{2}$ \\
Transmission efficiency & 0.93 \\
Air drag coefficient & 0.8 \\
Final drive ratio & 5.26 \\
\hline
\end{tabular}

since there is always an EM engaged in the powertrain, the wheel torque will not drop to 0 like AMT during the shift.

\section{Simulation and Discussion}

In this section, the energy consumption and shift performance of the eDMT and AMT are compared through the simulation process.

Fig. 8 Control strategy for mode shift

\subsection{Comparison of Energy Consumption}

window $(\Delta w)$, the dog clutch CU starts engaging from the middle position to the right position, and finally the speed of EM2 is synchronized with the target mode speed.

Phase 3: Lock-up phase Since both EM1 and EM2 are already engaged, their torques are adjusted coordinately to the setpoint according to the power distribution determined by the EMS.

It is noted that the torque compensation capability is limited by the peak torque of the EM. Therefore, it is difficult to maintain the wheel torque the same as that before the shift in some cases (e.g. continuous maximum torque). However,

The adapted world transient vehicle cycle (C-WTVC), a cycle for the application tests of heavy commercial vehicles, is employed to compare the energy consumption. The main parameters of the vehicle used for simulation are listed in Table 1.

The simulation results of eDMT using the mode shift map defined in Sect. 3.2 are displayed in Figs. 9, 10, 11. It is found that most operating points are located in the high-efficiency region of both EMs. With a driving cycle of $20.51 \mathrm{~km}$, the total energy consumption is $29.95 \mathrm{~kW} \cdot \mathrm{h}$.

A 4-speed AMT with the same motor and vehicle parameters as eDMT is used in the simulation. The 1st gear ratio of 


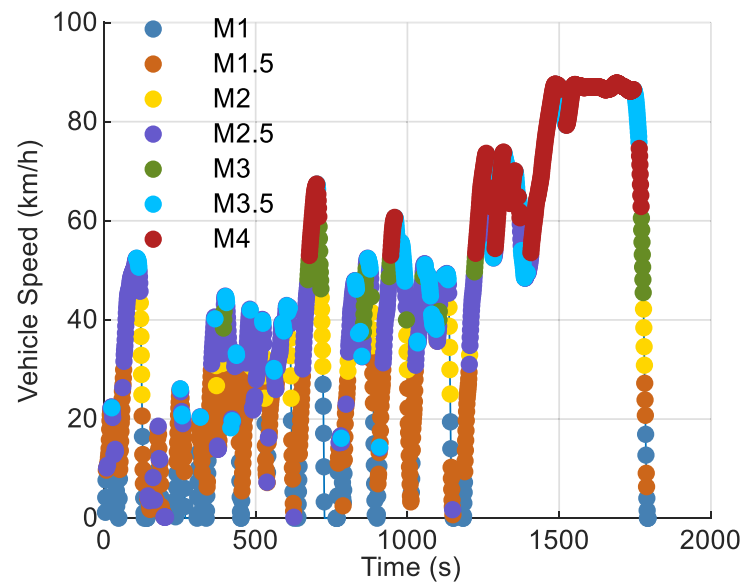

Fig. 9 C-WTVC cycle and the operating modes of the eDMT with runtime

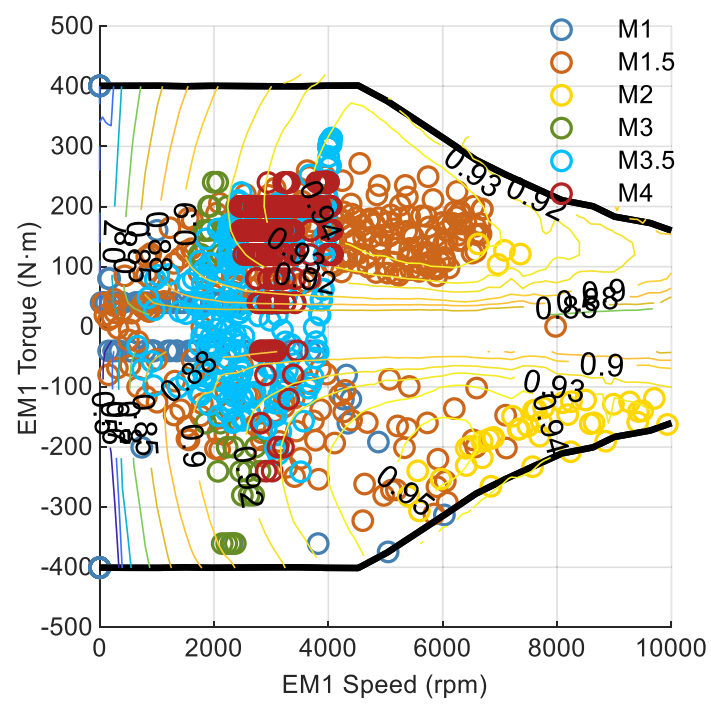

Fig. 10 EM1 operating points along the C-WTVC

AMT is determined regarding identical launch torque with the eDMT. The 4th gear ratio is obtained on the basis of the top speed capability. The proportional gear ratio step is applied in this AMT. Figure 12 shows the shift map of the 4-speed AMT defined by the same EMS proposed in Sect. 3. The simulation results of the vehicle with the 4-speed AMT are shown in Figs. 13 and 14. The total energy consumption of the 4-speed AMT is $31.36 \mathrm{~kW} \cdot \mathrm{h}$.

By comparing Figs. 10, 11, and 14, it is found that the proposed EMS can make the EM work in the high-efficiency area as much as possible. Furthermore, a lower energy consumption $(29.95 \mathrm{~W} \cdot \mathrm{h}$ versus $31.36 \mathrm{~kW} \cdot \mathrm{h}$ for the C-WTVC cycle of $20.51 \mathrm{~km}$ ) of the eDMT is reached through the reasonable power distribution between the two EMs.

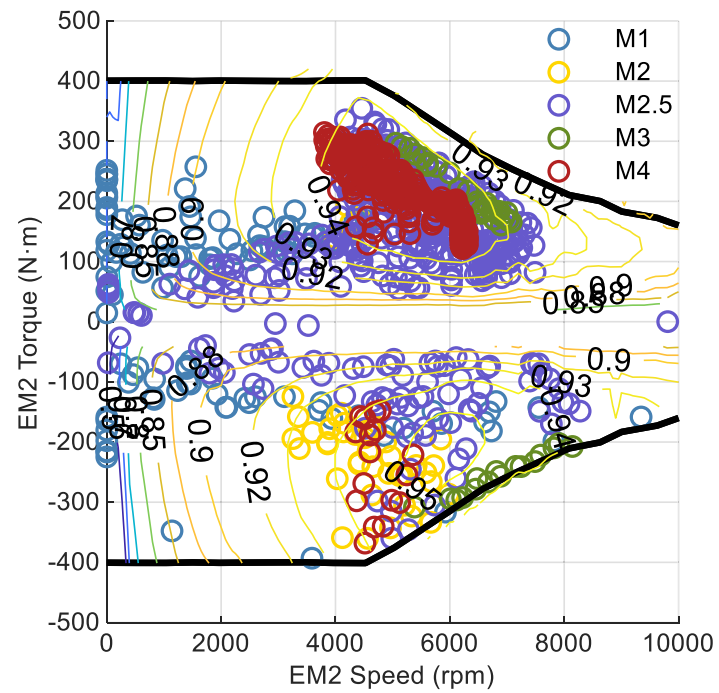

Fig. 11 EM2 operating points along the C-WTVC

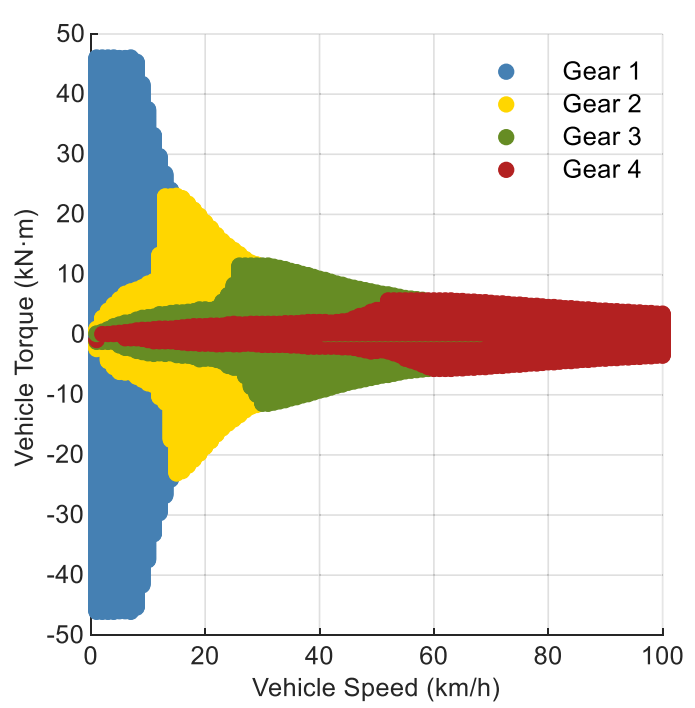

Fig. 12 Shift map of the 4-speed AMT

\subsection{Comparison of Shift Performance}

The simulation of shift performance is carried out for the vehicle accelerating at a $3 \%$ slope. Figure 15 shows the speed variation of EMs during the shift. It can be seen that two shifts occurred, one at about $10 \mathrm{~s}$ and the other at about $15 \mathrm{~s}$.

Figure 16 shows the torques variation of EMs during shift. It can be seen that only one EM's torque drops below 0 during the shift in eDMT. Since the torque of the other EM is very high, the torque shown in Fig. 16 does not increase to compensate for the torque loss during the shift. The EM 


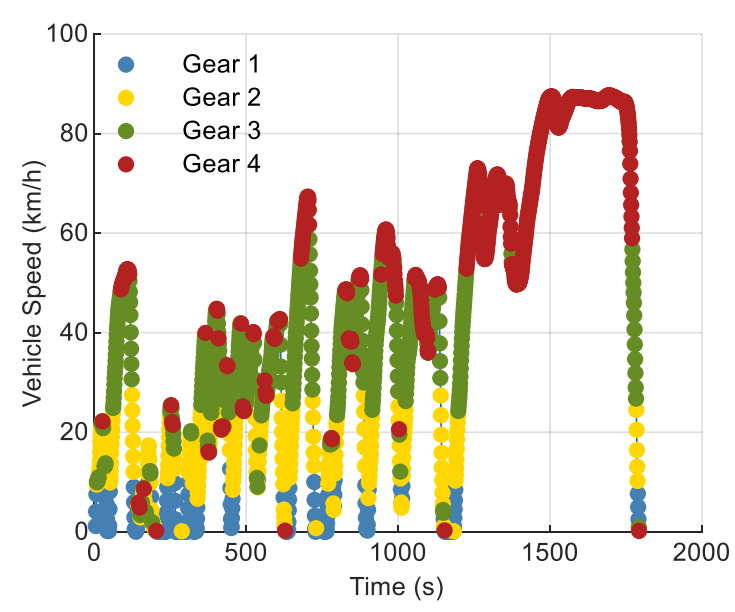

Fig. $13 \mathrm{C}$-WTVC and the operating gears of the 4-speed AMT along the cycle

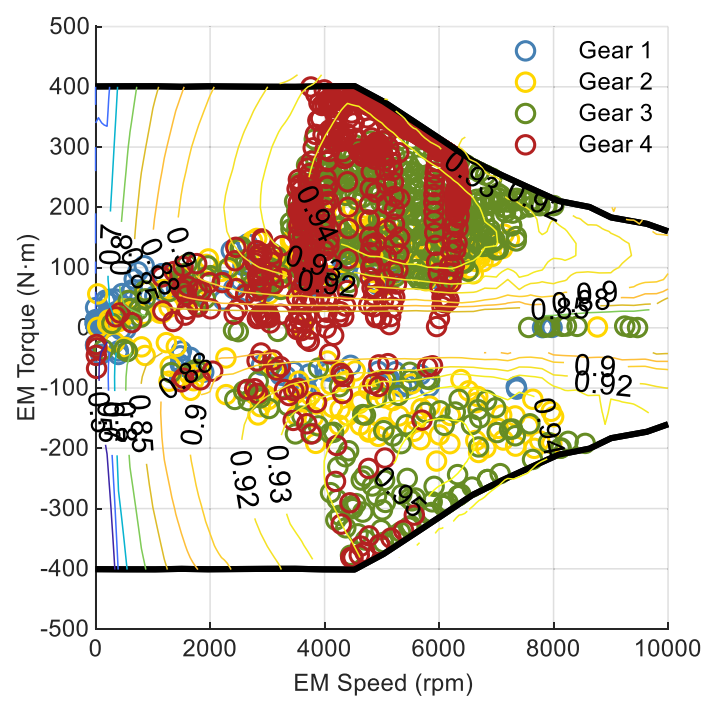

Fig. 14 EM operating points along the C-WTVC of the 4-speed AMT

torque of the AMT is also drastically reduced below 0 for speed regulation during shift.

Figure 17 shows the change of the vehicle acceleration. Obviously, the vehicle equipped with AMT suffers torque interruption and deceleration in the shifting process. When the vehicle is heavy and running on a slope, the deceleration caused by torque loss is very dangerous. Moreover, the large acceleration hole will leads to a poor subjective shift feeling of drivers. Compared with the acceleration of AMT, the vehicle using eDMT still accelerates in the shifting process. Although there is also a decrease in acceleration, the hole depth of eDMT is much smaller than that of the AMT.
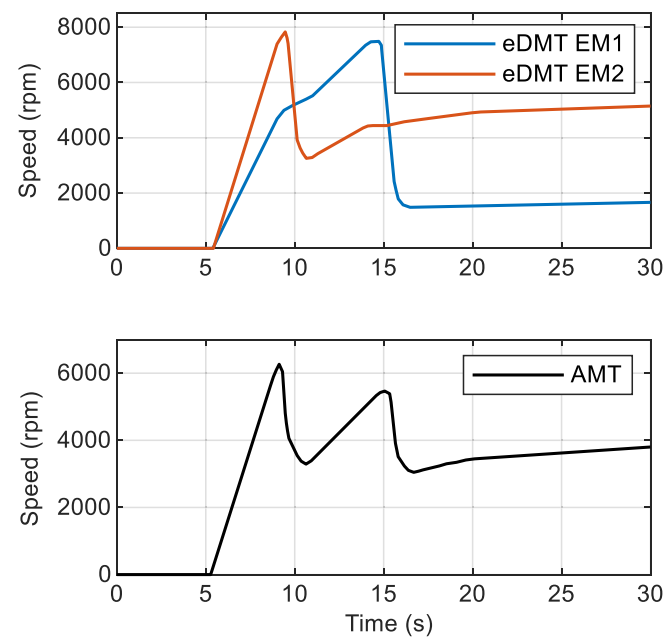

Fig. 15 The variation of EM speed during shift
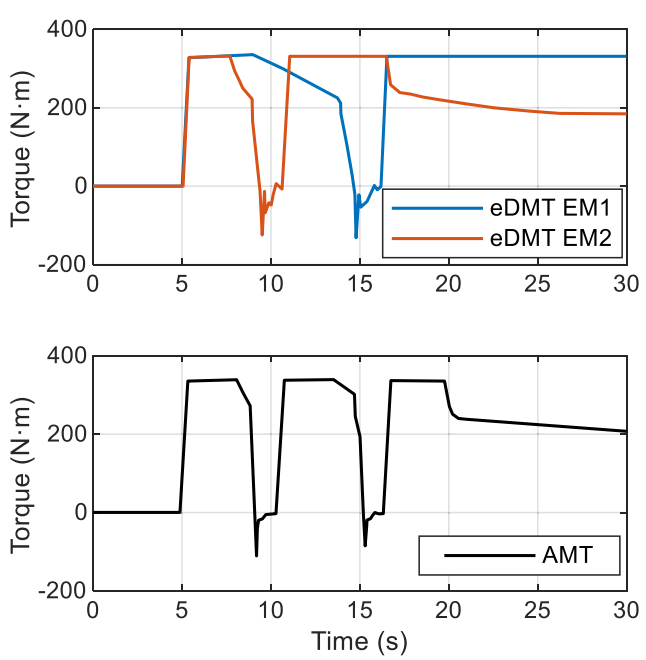

Fig. 16 EM torque variation during shift

Similarly, the dynamic performance of the vehicle with eDMT is much better than that with the AMT. As shown in Fig. 18, eDMT reaches $35 \mathrm{~km} / \mathrm{h}$ about $2.5 \mathrm{~s}$ earlier than the AMT. Particularly after the shift, the vehicle speed using AMT cannot keep up with the vehicle speed using eDMT.

The simulation results have verified that eDMT has better economic performance and dynamic performance than the conventional AMT in heavy commercial EVs. In addition, the power shift capability enables a advantages both in shift quality of eDMT. 


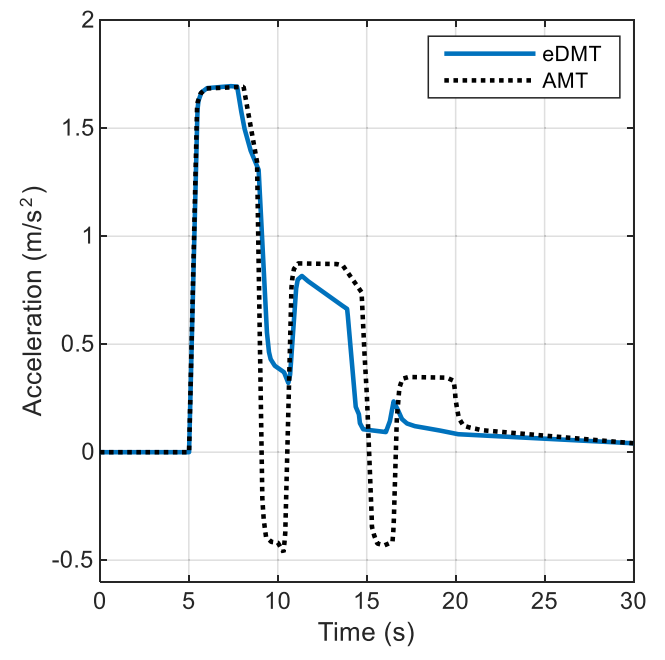

Fig. 17 Vehicle acceleration variation during shift

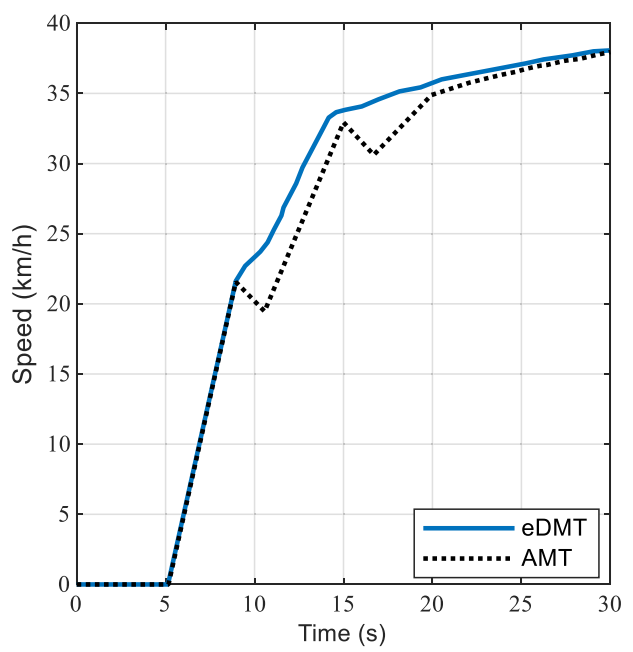

Fig. 18 Vehicle speed variation during shift

\section{Conclusions}

The main conclusions are drawn as follows:

(1) A novel electric dual motor transmission for heavy commercial vehicles is proposed. And the operating modes of the eDMT are analyzed.

(2) A rule-based EMS is advanced to design the mode shift map and the power distribution between two EMs with minimum energy consumption.

(3) A coordinated control strategy is developed to coordinately control the two EMs and the dog clutches for mode shift without torque interruption.
(4) The simulation results show that the eDMT is superior to the conventional AMT in terms of economic performance and dynamic performance in heavy commercial EVs.

The prototype will be tested in the future to verify the effectiveness of the designed mode shift map and the proposed coordinated control strategy.

Acknowledgements This work is financially supported by The 2025 Science and Technology Innovation Program of Ningbo "R\&D of Key Technologies for Electric Vehicle Range Extenders" (Grant No. 2019B10111), National Natural Science Foundation of China (NSFC, Grant No.52072018), Key Science and Technology Innovation Project of Shandong Province (Grant No. 2019JZZY010913) and Key Science and Technology Project of Guangxi Province (Grant No. AA19254013). The authors also acknowledge the support of the Beijing Key Laboratory for High-efficient Power Transmission and System Control of New Energy Resource Vehicle and the support of the Fundamental Research Funds for the Central Universities.

Open Access This article is licensed under a Creative Commons Attribution 4.0 International License, which permits use, sharing, adaptation, distribution and reproduction in any medium or format, as long as you give appropriate credit to the original author(s) and the source, provide a link to the Creative Commons licence, and indicate if changes were made. The images or other third party material in this article are included in the article's Creative Commons licence, unless indicated otherwise in a credit line to the material. If material is not included in the article's Creative Commons licence and your intended use is not permitted by statutory regulation or exceeds the permitted use, you will need to obtain permission directly from the copyright holder. To view a copy of this licence, visit http://creativecommons.org/licenses/by/4.0/.

\section{References}

1. Xu, X.Y., Dong, P., Liu, Y.F., et al.: Progress in automotive transmission technology. Automot. Innov. 1(3), 187-210 (2018)

2. Xu, X.Y., Chen, Z.F., Liu, Y.J., et al.: Enumerative optimization procedure for the gear train optimization problem of a two-speed dedicated electric transmission. Energies 10(9), 1362 (2017)

3. Zhao, B., Lv, C., Hofman, T., et al.: Design optimization of the transmission system for electric vehicles considering the dynamic efficiency of the regenerative brake. SAE Technical Paper 201801-0819 (2018)

4. Zhao, B., Lv, C., Zhang, J., et al.: Optimization of gear ratios for a two-speed transmission system of an electric passenger vehicle considering dynamical regenerative braking efficiency. SAE Technical Paper 2016-01-0436 (2016)

5. Zhang, L.P., Yang, L.Q., Guo, X.B., et al.: Stage-by-phase multivariable combination control for centralized and distributed drive modes switching of electric vehicles. Mech. Mach. Theory 147, 103752 (2020)

6. Dong, P., Liu, Y., Tenberge, P., et al.: Design and analysis of a novel multi-speed automatic transmission with four degrees-offreedom. Mech. Mach. Theory 108, 83-96 (2017)

7. Huang, J.F., Zhang, J.L., Huang, W., et al.: Optimal speed synchronization control with disturbance compensation for an integrated motor-transmission powertrain system. J. Dyn. Syst. -T. ASME 141(4), 041001 (2019) 
8. Xu, X., Liang, Y., Jordan, M., et al.: Optimized control of engine start assisted by the disconnect clutch in a P2 hybrid automatic transmission. Mech. Syst. Signal Pr. 124, 313-329 (2019)

9. Wang, S., Liu, Y., Wang, Z., et al.: Adaptive fuzzy iterative control strategy for the wet-clutch filling of automatic transmission. Mech. Syst. Signal Pr. 130, 164-182 (2019)

10. Sorniotti, A., Holdstock, T., Pilone, G.L., et al.: Analysis and simulation of the gearshift methodology for a novel two-speed transmission system for electric powertrains with a central motor. Proc. Inst. Mech. Eng. Part D J. Automob. Eng. 226, 915-929 (2012)

11. Dong, P., Wu, S., Guo, W., et al.: Coordinated clutch slip control for the engine start of vehicles with P2-hybrid automatic transmissions. Mech. Mach. Theory 153, 103899 (2020)

12. Zhu, X., Zhang, H., Xi, J., et al.: Optimal speed synchronization control for clutchless AMT systems in electric vehicles with preview actions. Paper presented at 2014 American Control Conference, Portland, Oregon, USA, 4-6 June 2014

13. Chen, H., Mitra, S.: Synthesis and verification of motor-transmission shift controller for electric vehicles. Paper presented at the 2014 ACM/IEEE International Conference on Cyber-Physical Systems. Berlin, Germany, 14-17 April 2014

14. Tian, Y., Ruan, J.G., Zhang, N., et al.: Modelling and control of a novel two-speed transmission for electric vehicles. Mech. Mach. Theory 127, 13-32 (2018)

15. Tseng, C.Y., Yu, C.H.: Advanced shifting control of synchronizer mechanisms for clutchless automatic manual transmission in an electric vehicle. Mech. Mach. Theory 84, 37-56 (2015)

16. Yu, C.H., Tseng, C.Y.: Research on gear-change control technology for the clutchless automatic-manual transmission of an electric vehicle. Proc. Inst. Mech. Eng. Part D J. Automob. Eng. 227, 1446-1458 (2013)

17. Gao, B.Z., Liang, Q., Xiang, Y., et al.: Gear ratio optimization and shift control of 2-speed I-AMT in electric vehicle. Mech. Syst. Signal Pr. 50-51, 615-631 (2015)

18. Mo, W.W., Walker, P.D., Fang, Y.H., et al.: A novel shift control concept for multi-speed electric vehicles. Mech. Syst. Signal Pr. 112, 171-193 (2018)

19. Mo, W.W., Walker, P.D., Zhang, N.: Dynamic analysis and control for an electric vehicle with harpoon-shift synchronizer. Mech. Mach. Theory 133, 750-766 (2019)

20. Roozegar, M., Angeles, J.: The optimal gear-shifting for a multispeed transmission system for electric vehicles. Mech. Mach. Theory 116, 1-13 (2017)
21. Chen, H.X., Tian, G.Y.: Modeling and simulation of gear shifting in clutchless coupled motor-transmission system. J. Tsinghua Univ. 56, 144-151 (2016)

22. Wang, X.Y., Li, L., He, K., et al.: Position and force switching control for gear engagement of automated manual transmission gear-shift process. J. Dyn. Syst. -T. ASME 140(8), 081010 (2018)

23. Wu, J.L., Liang, J.J., Ruan, J.G., et al.: Efficiency comparison of electric vehicles powertrains with dual motor and single motor input. Mech. Mach. Theory 128, 569-585 (2018)

24. Wu, J.L., Liang, J.J., Ruan, J.G., et al.: A robust energy management strategy for EVs with dual input power-split transmission. Mech. Syst. Signal Pr. 111, 442-455 (2018)

25. Wu, H.F., Walker, P.D., Wu, J.J., et al.: Energy management and shifting stability control for a novel dual input clutchless transmission system. Mech. Mach. Theory 135, 298-321 (2019)

26. Zhao, B., Lv, C., Hofman, T.: Driving-cycle-aware energy management of hybrid electric vehicles using a three-dimensional Markov chain model. Automot. Innov. 2(2), 146-156 (2019)

27. Sorniotti, A., Holdstock, T., Everitt, M., et al.: A novel clutchless multiple-speed transmission for electric axles. Int. J. Powertrains 2, 103-131 (2013)

28. Carlo, M.D., Mantriota, G.: Electric vehicles with two motors combined via planetary gear train. Mech. Mach. Theory 148, 103789 (2020)

29. Zhang, L.P., Li, L., Qi, B., et al.: Double-motor distributed drive vehicle high-speed stability electromechanical coupling control. J. Mech. Eng. 51, 29-40 (2015)

30. Zhang, L.P., Gu, D., Qi, B., et al.: Variable mode impact suppression method for electric vehicle centralized and distributed coupling drive system. J. Mech. Eng. 54, 165-176 (2018)

31. Zhang, L.P., Li, L., Qi, B., et al.: Parameters optimum matching of pure electric vehicle dual-mode coupling drive system. Sci. China Technol. Sc. 57, 2265-2277 (2014)

32. Liang, J.J., Yang, H.T., Wu, J.L., et al.: Power-on shifting in dual input clutchless power-shifting transmission for electric vehicles. Mech. Mach. Theory 121, 487-501 (2018)

33. Liang, J.J., Yang, H.T., Wu, J.L., et al.: Shifting and power sharing control of a novel dual input clutchless transmission for electric vehicles. Mech. Syst. Signal Pr. 104, 725-743 (2018) 